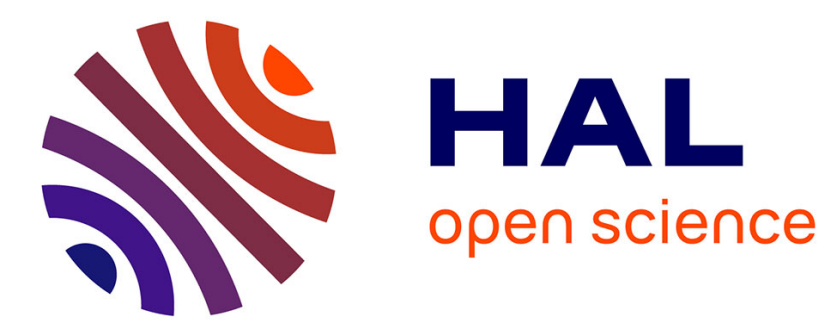

\title{
Air entrainment by a plunging jet: the dynamical roughness concept and its estimation by a light absorption technique
}

\author{
Laurent Davoust, Jean-Luc Achard, M. El Hammoumi
}

\section{- To cite this version:}

Laurent Davoust, Jean-Luc Achard, M. El Hammoumi. Air entrainment by a plunging jet: the dynamical roughness concept and its estimation by a light absorption technique. International Journal of Multiphase Flow, 2002, 28, pp.1541-1564. 10.1016/S0301-9322(02)00040-X . hal-00182324

\author{
HAL Id: hal-00182324 \\ https://hal.science/hal-00182324
}

Submitted on 17 Feb 2020

HAL is a multi-disciplinary open access archive for the deposit and dissemination of scientific research documents, whether they are published or not. The documents may come from teaching and research institutions in France or abroad, or from public or private research centers.
L'archive ouverte pluridisciplinaire HAL, est destinée au dépôt et à la diffusion de documents scientifiques de niveau recherche, publiés ou non, émanant des établissements d'enseignement et de recherche français ou étrangers, des laboratoires publics ou privés. 


\title{
Air entrainment by a plunging jet: the dynamical roughness concept and its estimation by a light absorption technique
}

\author{
L. Davoust ${ }^{\mathrm{a}, *}$, J.L. Achard ${ }^{\mathrm{a}, 1}$, M. El Hammoumi ${ }^{\mathrm{b}, 2}$ \\ ${ }^{\text {a }}$ Laboratoire des Ecoulements Géophysiques et Industriels (LEGI), B.P. 53, 38041 Grenoble Cedex 09, France \\ ${ }^{\mathrm{b}}$ Faculté des Sciences et Techniques Fès Sä̈ss, Département de Physique, Laboratoire de Mécanique Appliquée, \\ B.P. 2202, Fès, Morocco
}

Air entrainment by plunging jets and the consequent production of bubbles play an increasing role in industrial and environmental applications. Generally, air entrainment can be seen as the consequence of two complementary mechanisms: (1) the interfacial shear along the liquid jet interface which drags down an air boundary layer and (2) the air entrapment process at the point of impact of the plunging jet with the receiving pool. The latter process is usually dominated by growing interfacial disturbances travelling on the jet. The great variety of these disturbances is represented by the concept of dynamic interfacial roughness of which a definition is provided and justified. To measure this quantity, an easy-to-use optical technique is designed. The dynamic interfacial roughness and the ability to entrap air by a plunging jet are found to depend on the hydrodynamical noise applied to the jet. The optical technique is presented as a very simple tool to optimally devise the internal design of nozzles and then, to control air entrainment.

Keywords: Interface; Roughness; Free surface turbulence; Plunging jet; Liquid jet; Air entrainment; Entrapment; Laser; Light absorption

\footnotetext{
${ }^{*}$ Correponding author. Tel.: +33-4-76-82-50-38; fax: +33-4-76-82-52-71.

E-mail addresses: laurent.davoust@hmg.inpg.fr (L. Davoust), jean-luc.achard@hmg.inpg.fr (J.L. Achard).

${ }^{1}$ Tel.: +33-4-76-82-50-44; fax: +33-4-76-82-52-71.

${ }^{2}$ Tel.: +212-55-60-29-53; fax: +212-55-60-82-14.
} 


\section{Introduction}

Air entrainment is one of the widespread phenomena encountered in nature as well as in the industrial processes; depending on the situation under consideration, it is considered as welcome or unwelcome.

In many engineering and environmental flows (e.g. on chutes, spillways and breaking waves, respectively), aeration by air entrainment is a welcome effect since it reduces damages due to cavitation (Chanson, 1992) or it enhances mass transfers producing larger interfacial area densities (Chanson and Cummings, 1992). For instance, plunging waves in the ocean entrap large pockets of air which break up into bubble plums. When these bubbles collapse at the surface of the ocean they enhance an efficient exchange of mineral salts and atmospheric gases with the atmosphere.

In many chemical reactors (food processing, iron casting), a plunging jet impinging in a pool or the buffeting of the bulk fluid give rise to bubbly plums, which are responsible for foaming. This foaming is often considered as an inopportune and unwelcome effect. The foam is usually timestabilised by the addition of surfactants, which prevent the interstitial liquid film, wedged between the bubbles, from a quick draining. Two strategies are able to jam this foaming. The first one requires physicochemical concepts that make the stability of the interstitial film to vanish. The second one consists in damping the interfacial disturbances that could develop on the jet and contribute to the amount of air entrapped. This applies upstream from the impact of the plunging jet with the liquid pool. In view of the diversity of these interfacial disturbances, the dynamical roughness is proving to stand as a convenient and unifying concept.

In addition, we present an optical technique, based on light absorption, which allows for the dynamical roughness of a liquid jet to be measured accurately at the nozzle of a supplier injector. A review of the literature reveals that several outstanding issues, which demand to be enlightened by experimental evidence, may be resolved by the use of this technique.

\section{Relevance of the initial interfacial roughness concept}

In this paragraph, we discuss the relevance of the initial interfacial roughness of the jet to the theory available in the literature. Its precise definition and its measurement serve several purposes. It can help to formulate better correlations quantifying air entrainment; specific physical mechanisms involved in air entrapment processes can be isolated and measurements of the initial roughness can be employed to optimise the design of nozzles producing very smooth high-speed jets.

\subsection{Weakness of present dimensional correlations providing air entrainment}

Air entrainment by plunging jets impinging free surfaces has received considerable attention (see e.g. Van de Sande and Smith, 1976; McKeogh and Ervine, 1981; Iciek, 1982; Ervine and Falvey, 1987). McKeogh and Ervine (1981) first put forward three typical scenarii of the air entrapment process at the impinging point in a liquid pool (Fig. 1). The first scenario, here referred to as scenario \#I, is concerned with laminar jets. The jet penetrates deeply into the pool and 


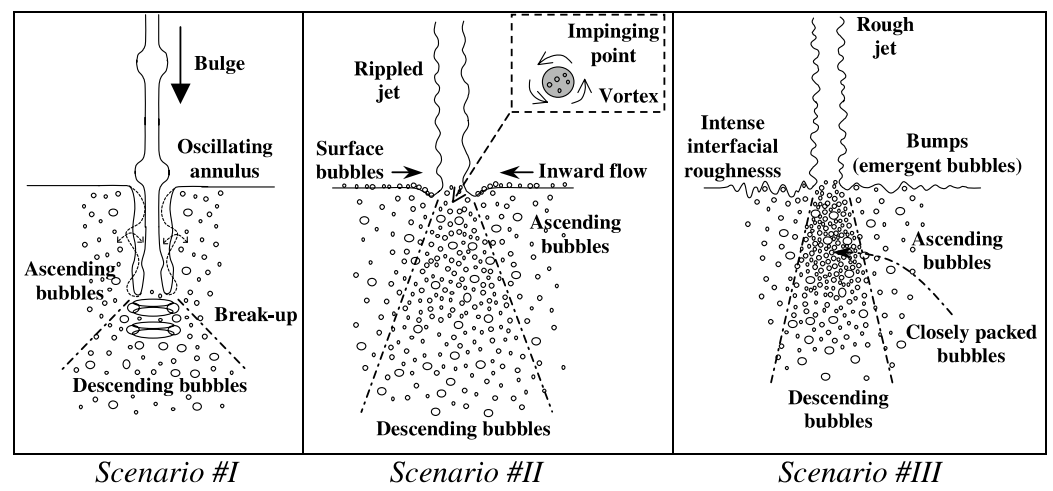

Fig. 1. Typical scenarii of air entrainment depending on the Reynolds number of the plunging jet (after McKeogh and Ervine, 1981; Ohl et al., 2000).

gives rise to an annular air cavity beneath the free surface. The inner interface of this air cavity extends the jet interface whereas the facing outer interface connects the bottom of the jet to the free surface of the pool. The envelope of the air cavity is found to oscillate much. Any interfacial disturbance like a bulge travelling on the jet makes the cohesion of the air cavity unstable, provoking its puncture at one or several locations: the thickness of the cavity is comparable to the amplitude of such a disturbance (see e.g. pioneering observation realised by Lin and Donnelly (1966), performed by way of photographs, see also a recent modelling carried out by Zhu et al. (2000)). All the secondary pockets which detach from the air cavity are themselves submitted to further Rayleigh-Taylor instabilities and disintegrate into bubbles to yield a bubbly plum. If the jet velocity is slow enough, then the jet interface is perfectly smooth and none bulge travels on the jet. Consequently, no air entrapment occurs within the pool. It is not obvious to give an exact value of the critical jet Reynolds number from which this scenario \#I begins to occur because, as will be explained later, the internal geometry of the injector turns out to be a prior parameter.

A second scenario, here referred to as scenario \#II, is found during the transition of the jet from a laminar flow to turbulence. An intermittent vortex is observed at the impinging point; the free surface of the pool deepens around the jet. The dip is explained by the increasingly axial momentum driven by the jet and also by the axial flow of the air boundary layer which surrounds the jet.

The third scenario, referred to as scenario \#III, is related to an established turbulent plunging jet. Both the surface of the jet and the surface of the pool are shaken up at the vicinity of the impact point so that many bubbles are efficiently generated.

The review by Bin (1993) about air entrainment by plunging jets gathers the whole of the available correlations established to predict either, the onset from which air entrainment occurs (say, the critical velocity of the jet), or the flow rate of air carryunder, or the typical diameter distributions within the bubbly plum which expands down to the inner of the pool.

Identifying the above scenarii can be used to draw out semi-empirical laws. Bin (1993) has compared the various models of the gas flow rate $\Phi_{\mathrm{a}}$ carried by a plunging jet (Mc Carthy et al., 1970; Burgess et al., 1972; Van de Sande and Smith, 1973, 1976; Lara, 1979; McKeogh and Ervine, 1981; Sene, 1988) with the experimental data of the literature. When jets are viscous (such as oil jets), some of these empirical models seem to be in fair agreement with experiments; 
nevertheless, it remains very difficult to draw any conclusion about the validity of one particular model among others since only some of them propose a non-dimensional analysis (see e.g. Van de Sande and Smith, 1976; Kusabiraki et al., 1990a,b).

The situation is worse when jets are weakly-viscous (water jets typically). As pointed out by Bin (1993) and other authors before (McKeogh and Ervine, 1981), the internal geometry of the injector supplying the jet becomes also of prior importance: the amount of air carryunder is observed to change drastically depending on how the nozzle is straight, convergent or divergent, depending on how the internal walls of the injector are smooth or slightly corrugated, how the jet is sensitive to any perturbations from the outside (aerodynamical fluctuations, external vibrations ...). In other words, any residual hydrodynamical noise applied upstream from the exit of the injector is able to change dramatically the flow rate $\Phi_{\mathrm{a}}$. This point is clearly demonstrated at the end of this paper. Consistently, as soon as the liquid jets under consideration are weakly viscous, there is an increasing difficulty to find one model that fits all the experimental measurements available in literature. Moreover, as already mentioned, only some authors (Kusabiraki et al., 1990a,b) put forward a non-dimensional analysis which allows to offer actually a convenient basis to compare with the experimental data. Note that the situation is quite different for the studies concerned with the stability of a jet submitted to a strong aerodynamic interaction; the non-exhaustive list of authors which follows is enough to be convinced (Weber (1931); Grant and Middleman (1966); Fenn and Middleman (1969); Phinney (1973); Sterling and Sleicher (1975); Lafrance (1977); Reitz and Braco (1982); Torpey (1989)). This literature shows how to deal with the (weak) coupling between the liquid jet and the surrounding flow of air carryunder by way of the so-called entrainment Weber number, $W e_{\mathrm{n}}$. This non-dimensional number can also be used in our problem to quantify the flow rate $\Phi_{\mathrm{a}}$.

These comments enlighten our experimental investigation on cylindrical injectors; their length and diameter were varied over a large range together with the viscosity of the liquid involved in the plunging jet (see a companion paper by El Hammoumi et al. (in press)). In this experimental study, our purpose was to insure well-defined exit conditions which were simply those of a fullydeveloped velocity profile in a smooth circular tube. From a systematic non-dimensional analysis of the involved physical mechanisms, two expressions were made evident, which apply to the entrainment Weber number, here defined as $W e_{\mathrm{n}}=\left(\rho_{\mathrm{a}} V_{\mathrm{a}}^{2} d\right) / \sigma$. These expressions, recalled hereafter, are established in details by El Hammoumi et al. (in press) and hold whether the jet is either laminar or turbulent:

$$
\begin{aligned}
& W e_{\mathrm{n}}=1.37 \times 10^{-2} W e_{\mathrm{j}}^{1.34} \widetilde{H}^{1.77} Z^{2.59} \mathrm{Fr}^{0.89} \quad \text { (turbulent regime) }, \\
& W e_{\mathrm{n}}=5.85 \times 10^{-9} \mathrm{We}_{\mathrm{j}}^{0.10} \widetilde{H}^{1.29} Z^{-1.47} \mathrm{Fr}^{0.32} \quad \text { (laminar regime) } .
\end{aligned}
$$

The following non-dimensional numbers are subsequently involved:

- the classical Weber number, $W e_{\mathrm{j}}=\left(\rho_{1} V_{1}^{2} d\right) / \sigma$, which plays a key-role into dynamics of the interfacial stability modes,

- the Öhnesorge number, $Z=v_{1}\left(\rho_{1} / \sigma d\right)^{1 / 2}$, which takes into account the relaxation of the viscous stress immediately at the exit of the injector,

- the Froude number, $F r=V_{\mathrm{j}}^{2} / g d$, which, in this particular free-surface problem, allows to take into account the stabilising role of the gravity $g$, 
- the non-dimensional falling height of the jet, $\widetilde{H}=H / d$ (aspect ratio of the distance between the exit of the injector and the impact point to the jet diameter), which characterises the geometry of the problem.

Not surprisingly, the non-dimensional numbers $W e_{\mathrm{j}}$ and $Z$ are also directly involved in the literature devoted to the stability of jets submitted to a strong aerodynamic interaction.

The physical characteristics are taken into account through the density and the viscosity ratios, respectively, $\varepsilon=\rho_{\mathrm{a}} / \rho_{1}$ and $K=v_{\mathrm{a}} / v_{1}$. The variables $\rho_{\mathrm{a}}, v_{\mathrm{a}}, V_{\mathrm{a}}, \rho_{\mathrm{l}}, v_{\mathrm{l}}, V_{1}, d, \sigma$ denote the density, the molecular viscosity, the typical velocities of the air and the liquid jet, the diameter of the jet and the surface tension.

The non-dimensional expressions of the entrainment Weber number compare favourably with our experimental data and also with those obtained by Van de Sande and Smith (1976) and Bin $(1984,1988)$ except when the jet Reynolds numbers reaches very large values (larger than 50000$)$ : both the jet and the free surface of the pool become fully-turbulent. In this particular situation, a first explanation relies on the jet momentum-induced agitation at the free surface of the receiving pool which can play an increasing role in the air entrapment process as the jet turbulence arises; such a surface agitation stands as an unknown and uncontrollable parameter (scenario \#III). A second explanation is related to the technology of the injectors itself: the aspect ratio of the nozzle and the roughness of the inner walls are rarely controlled.

Finally, stability theories developed up to now do not succeed in describing air entrainment during the transition since the jet is highly sensitive to all the external disturbances experienced by the liquid flow from the entry to the exit of the injector. These disturbances directly modulate the initial level of the interfacial jet roughness at the exit of the injector. On the other hand, empirical models based on the impact interfacial jet roughness alone (McKeogh and Ervine, 1981) are neither able to predict the flow rate $\Phi_{\mathrm{a}}$ during the transition. Finally, it can be stated that the initial jet roughness - as an indicator of the hydrodynamical forcing - is worth being actually considered for further refinement of any spatial stability analysis devoted to liquid jets. From such refined analysis, one could expect a more relevant non-dimensional correlation able to predict air entrainment even when the plunging jet is weakly viscous.

\subsection{Air entrainment as viscous versus potential flows}

This paragraph aims at demonstrating how the non-intrusive measurement of the jet roughness above the impinging point is a way to clarify a fundamental issue which can be illustrated by the following closely linked questions:

(i) What are the physical mechanisms actually involved in the air entrainment process?

(ii) Are these physical mechanisms purely potential or viscous in nature?

According to Mc Carthy et al. (1970), air entrainment can be modelled as a purely geometric process which integrates the combined effects of the interaction between the interfacial disturbances on the jet and the upper free surface of the receiving pool. The empirical model developed by McKeogh and Ervine (1981), also supported by geometrical considerations, focuses on 
interfacial mechanisms; two contributions are identified: the geometrical undulations of the interface - each of them being able to entrap and carry an elementary volume of air-and the flow of the air boundary layer at the vicinity of the interface. Consequently, two physical mechanisms are simultaneously involved in the air entrainment process: the aforementioned air entrapment at the impinging point, which comes under a purely potential hydrodynamics, and the tangential viscous stresses dragging down an air boundary layer along the jet. To our knowledge, the specific ability of each of these two mechanisms in contributing to air entrainment has never been clearly elucidated. Hence, the empirical way by which the model of McKeogh and Ervine (1981) takes into account the air boundary layer is questionable.

The measurement of the interfacial roughness turns out to be a convenient tool to investigate experimentally the validity of current modelling on the air entrapment process. The great majority of the models recently developed in the literature assume that air entrapment is governed by potential flows (scenario \#I, see e.g. Prosperetti and Oguz, 1997; Oguz, 1998; Zhu et al., 2000; Ohl et al., 2000). Only certain (Lezzi and Prosperetti, 1991) address the question of the stability of an annular air sheet representing the extension of the jet-driven air boundary layer beneath the free surface of the pool. This (thin) annular air sheet is able to destabilise further into bubbles within the pool (see e.g. the courtesy of Duncan displayed in the paper by Lezzi and Prosperetti (1991)). In this particular scenario, say, scenario \#I-bis, there is no need for bulges formation since shearinduced instabilities (Kelvin-Helmholtz waves or small viscous waves) may pinch-off the underwater air sheet. As far as we know, there is no experimental evidence of this scenario. A full experimental evidence of the theory of Lezzi and Prosperetti, would require:

1. to demonstrate the dependence of the stability thresholds on the capillarity number and the Reynolds number defined by the jet velocity, the thickness of the underwater air sheet, the density and the kinematic viscosity of the air entrained,

2. to get a perfectly smooth liquid jet even at a very large Reynolds number $\left(R e_{\mathrm{j}} \sim 10^{4}-10^{5}\right)$.

The optimisation procedure presented below (Section 2.3) complies with the latter requirement.

Until now, only the scenario \#I has been the attempt of a modelling based on purely potential flows (see e.g. Ohl et al., 2000). Air entrainment is thus described in terms of local air entrapment mechanisms at the impinging point. In this prospect, concluding experiments have been performed by Zhu et al. (2000) who generated one travelling bulge (interfacial disturbance) on the plunging jet by a sudden change of the flow rate at the nozzle. The shape of the travelling bulge was carefully detected (CCD camera) and the comparison with their potential flow model showed good agreement. Nevertheless, such modelling remains still far from the reality of scenario \#I since the interfacial disturbances are not due to a sudden change of the input flow rate but rather due to the inherent jet instability. Ideally, it could be of prior interest to control the jet instability by exciting travelling interfacial waves with a given symmetry (axial or non-axial) and a given periodicity. For this purpose, the internal design of the injector must be carefully devised. This can be achieved taking advantage of an annular contraction, managed inside the injector, whose aperture can be tuned so that initial relaminarisation of the liquid jet is more or less promoted (El Hammoumi et al., 1999). This parametrical control of the initial jet quality from a variablegeometry nozzle is technically different from the technique developed by Bonetto and Lahey (1993). 


\subsection{The initial interfacial roughness as a control parameter}

The above theoretical considerations reveal the fundamental interest to accurately control the stability of a weakly-viscous jet in order to render it perfectly smooth even at a large Re-number, $R e_{\mathrm{j}} \sim 10^{4}-10^{5}$ or in order to enhance the arising of particular interfacial waves on the jet. There is the aim at stating definitely on the purely potential character of air entrainment. There is also the practical demand for reducing the amount of air carryunder in many industrial processes. As pointed out by Oguz (1998), a liquid jet experiences the development of a mixing boundary layer immediately from the nozzle. At large Weber number, the boundary layer becomes turbulent and is typically accompanied by the onset and the growing of travelling instabilities. Both the temporal and the spatial growing of unstable modes along the jet are sensitive to the magnitude of the hydrodynamical noise available at the outlet of the nozzle (Wu et al., 1995b). In other words, the liquid jet is an amplifier of any input hydrodynamical perturbations. The overall Fig. 2 illustrates this consideration.

Subsequently, the initial jet roughness stands as an important control parameter to handle since it represents the signature of the interfacial agitation on the jet. It is thus worth developing a nonintrusive technique to measure the jet roughness. Until now, the ability of a turbulent plunging jet to drag surrounded air has mainly been investigated experimentally by use of visual information of the interfacial turbulence around the impact point (high-speed video) or by LDA/PDA measurements performed on the bubbly plum within the pool (Bonetto and Lahey, 1993). These techniques are a very indirect way to give an insight into the level of disturbances applied to the jet. As far as we know, no technique devoted to the direct measurement of the interfacial

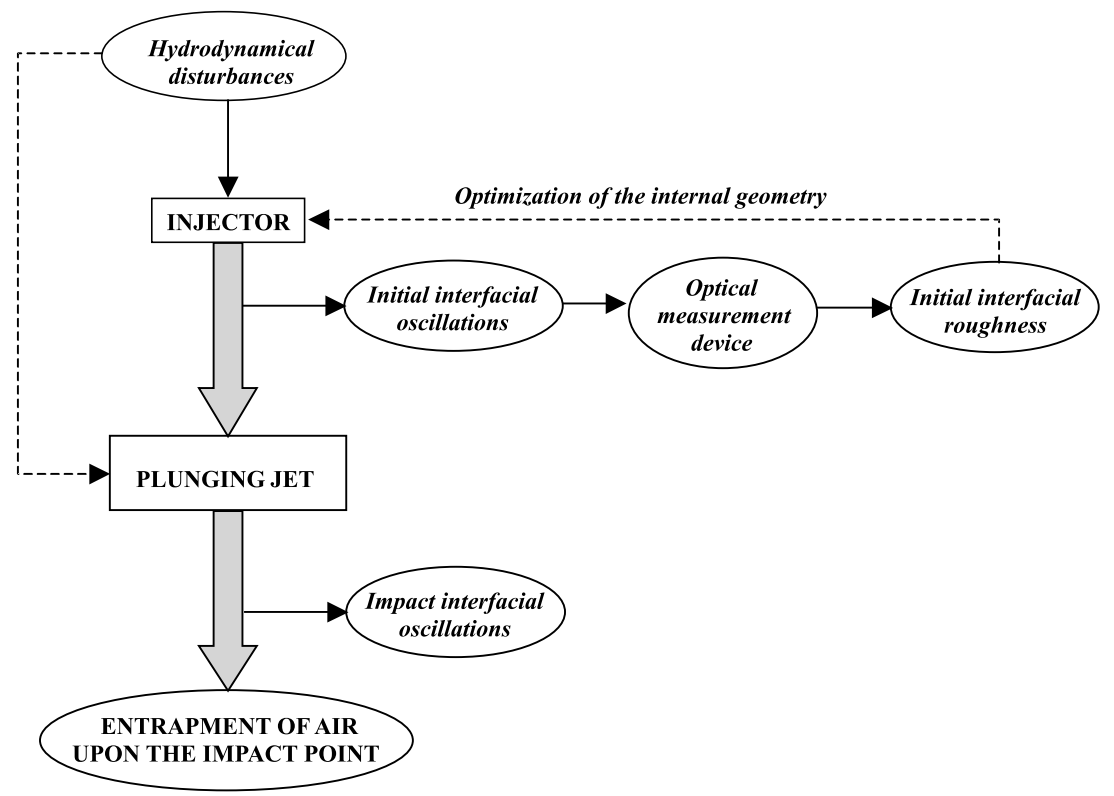

Fig. 2. The plunging jet as an amplifier of hydrodynamical disturbances. 
roughness is available in the literature though it could be very useful e.g. to investigate nearsurface turbulence. ${ }^{3}$

\section{Measurement of the initial interfacial roughness}

The diagnostic of the jet quality just after the nozzle requires the use of an optical technique which allows for an accurate quantification of the level of the interfacial roughness. The optical technique proposed here is by far much simpler to develop than the usual imaging techniques, which are time-consuming and too limiting in speed. The basic principle is to gain advantage from the interaction of a laser beam with a liquid jet. Such an interaction has already been employed for the study of a capillary jet (Cossali and Coghe, 1993) by using the transmission and subsequent deviation of a focused laser beam by the jet. Here, the optical technique that we put forward is simpler since it is based on light absorption. This optical technique has proved to be accurate in the different context of the thickness measurement of wavy liquid films (Mouza et al., 2000).

The physical principle of the optical technique here proposed is somewhat elementary: basically, the ratio between the light intensity occulted by the presence of the jet and the total delivered light intensity gives a valuable indication of the interfacial deformation of the liquid jet which can be considered as a black screen with respect to an incident laser beam. At the edge of the liquid jet, every ray of light which intercepts the liquid is absorbed in view of mere geometrical considerations (Fig. 3a). To illustrate this phenomenon more precisely, consider previous studies led on the scattering of light by a transparent particle of typical diameter $d$, much larger than the wavelength of the incident laser beam $\lambda_{0}$ (Fig. 3b). When a light ray interacts with a particle $\left(\lambda_{0} \ll d\right)$, its subsequent intensity falls off along the incident direction because of three mechanisms: reflection, refraction and diffraction. The deviated part of the light due to reflection and refraction is scattered all away the particle whereas the diffracted part is concentrated within a small cone with respect to the initial propagation direction. This diffraction cone is known to be characterised by a small angle $\theta_{\mathrm{d}}$ (Obha and Itoh, 1978),

$$
\theta_{\mathrm{d}} \approx 5.24 \frac{\lambda_{0}}{d}
$$

Thus, in the case of the liquid jet, the laser beam is expected to divide into:

(1) an occulted part which relates to the light reflection and refraction,

(2) and a non-occulted part which includes the diffraction cone.

The technique, which consists in sending directly the non-occulted part of the laser light to a photoreceptor located behind the jet, is not recommended since it is important to remove the diffraction cone. Consequently, the measurement set-up here involved is made up of a laser source, a non-focal optical system, a beam splitter plus a mirror, and finally, a photoreceptor (Fig. 4). The mirror located behind the liquid jet allows for the removal of the diffracted light which

\footnotetext{
${ }^{3}$ In this respect, Dabiri (1999) has recently investigated how near-surface turbulence and the RMS level of the interfacial roughness can be correlated.
} 

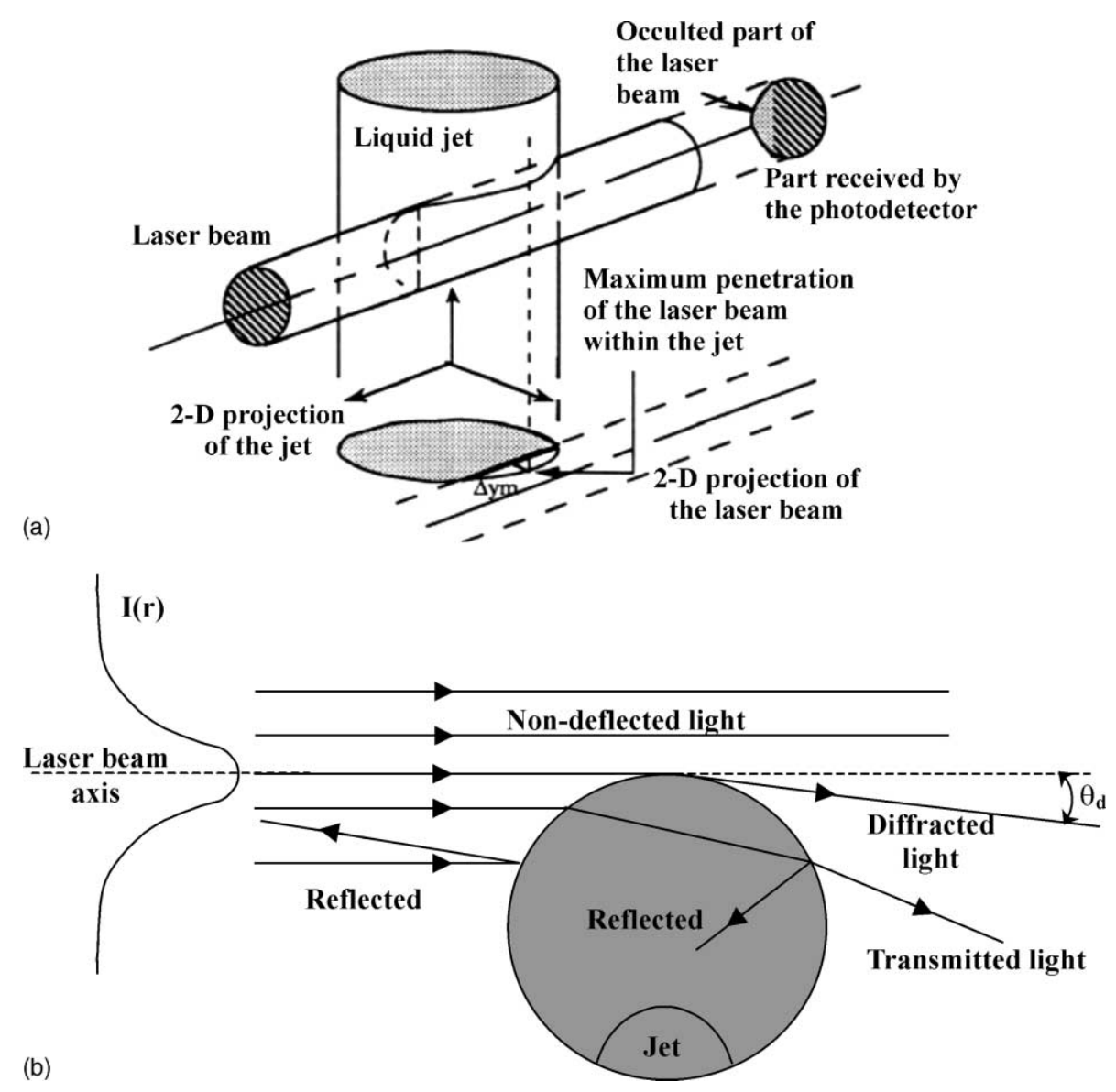

Fig. 3. (a) A 3-D schematic of the light absorption at the edge of the water jet. (b) Scattering of the incident laser light at the edge of the jet, as sketched.

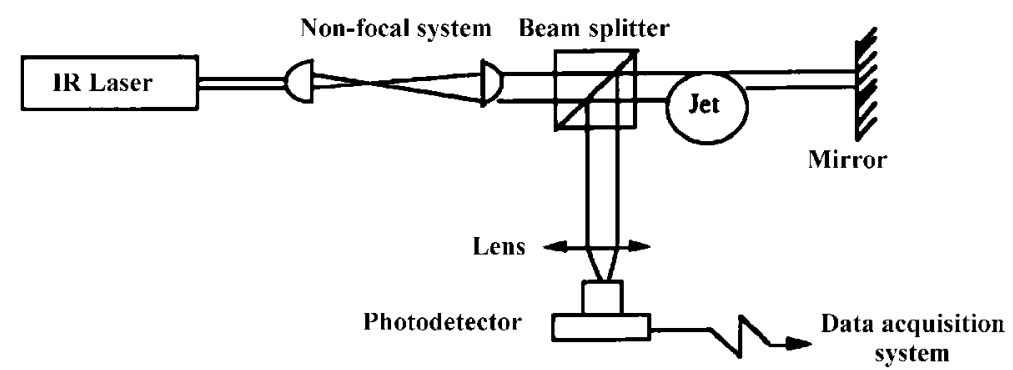

Fig. 4. The light absorption technique.

reflects not exactly perpendicularly onto its surface, comes back to the liquid jet and is definitely deflected. Much care is taken to align the mirror perpendicularly to the optical axis and to select and send back only the relevant part of the non-occulted light to the beam splitter. 
As a laser source, we make use of an ordinary He-Ne laser (power $5 \mathrm{~mW}$ ) whose beam is expanded by a factor of 4 with a non-focal system (two ordinary convergent lenses). For such an ordinary laser source, it is worthwhile to checking whether the intensity distribution along a diameter of the laser beam cross-section is both Gaussian and axisymmetric: it turns out that it is not so easy to lay one's hand on an ordinary laser source free of any transversal modes. Any slight defect of verticality or parallelism on the two mirrors located at the two ends of the resonant laser cavity yields transversal modes $\operatorname{TEM}_{p l q}(p, l \neq 0)$ on the light intensity distribution. The axisymmetry holds only if the azimuthal modes are vanishing $(l=0)$. The Gaussian distribution breaks up whether any radial mode is present $(p \neq 0)$. Local measurements of the light intensity $I(r)$ performed with fibre-optics along the radius of a cross-section of the expanded laser beam show unambiguously that axisymmetry was not well-achieved with the laser source involved in the present study. In such a situation, the light absorption due to a larger and larger penetration of the laser beam in the liquid jet cannot be analytically predicted in a straightforward way (Appendix A). For this technical reason and for the reason that we were not particularly aimed at investigating the details of the interfacial waves propagating on the jet, a direct in situ calibration of the optical set-up was preferred in the present study.

\subsection{Calibration of the light absorption technique}

When the set-up is stabilised (laser emission and electronics), the optics is calibrated with a smooth water jet (low Reynolds number jet): the purpose is to connect unambiguously the transversal penetration $\Delta Y_{\mathrm{m}}$ of the laser beam into the liquid jet to the level of light absorption (Fig. 9, Appendix A). The level of light absorption is defined as:

$$
A\left(\Delta Y_{\mathrm{m}}\right)=\frac{V\left(\Delta Y_{\mathrm{m}}\right)}{V_{0}}=\frac{I\left(\Delta Y_{\mathrm{m}}\right)}{I_{0}},
$$

where $V\left(\Delta Y_{\mathrm{m}}\right)$ and $V_{0}$ denote the output voltages as measured by the data acquisition system and are related to the light energies $I\left(\Delta Y_{\mathrm{m}}\right)$ and $I_{0}$ obtained when the laser beam is, respectively, occulted and not occulted. Before any experiment, the nozzle and the liquid jet were carefully aligned with gravity by means of a plumb line and the laser beam was set as horizontal as possible. At the beginning of the calibration procedure, the laser beam is located at the edge of the liquid jet: $\Delta Y_{\mathrm{m}}=0$. It follows that $A(0)=1$ and the quantity $A\left(\Delta Y_{\mathrm{m}}\right)$ decreases monotonically as the laser beam penetrates the plunging jet except at the middle point of the curve where a bump is made evident (Fig. 5): this local increase of light energy is attributed to the few light rays at the centre of the laser beam which are not significantly deviated by the jet interface. The normal to the jet interface at this exact location can be thought of as not only coplanar but also perfectly aligned with the optical axis. The calibration is carried out through a step by step displacement of the optical set-up. At each location of the laser beam, the DC value $V\left(\Delta Y_{\mathrm{m}}\right)$ of the voltage delivered by the photodetector is stored. This (transversal) displacement across the liquid jet is usually set with a constant step of $1 / 10 \mathrm{~mm}$ except at the vicinity of the air/water interface. In order to get an accurate calibration of the set-up, it is of prior importance to pinpoint the exact location for which both, the edge of the laser spot and the edge of the smooth water jet, are touching lightly each others. This exact location indicates the beginning of the calibration procedure. But the transversal light distribution across the laser beam does not exhibit any strong intensity discontinuity 


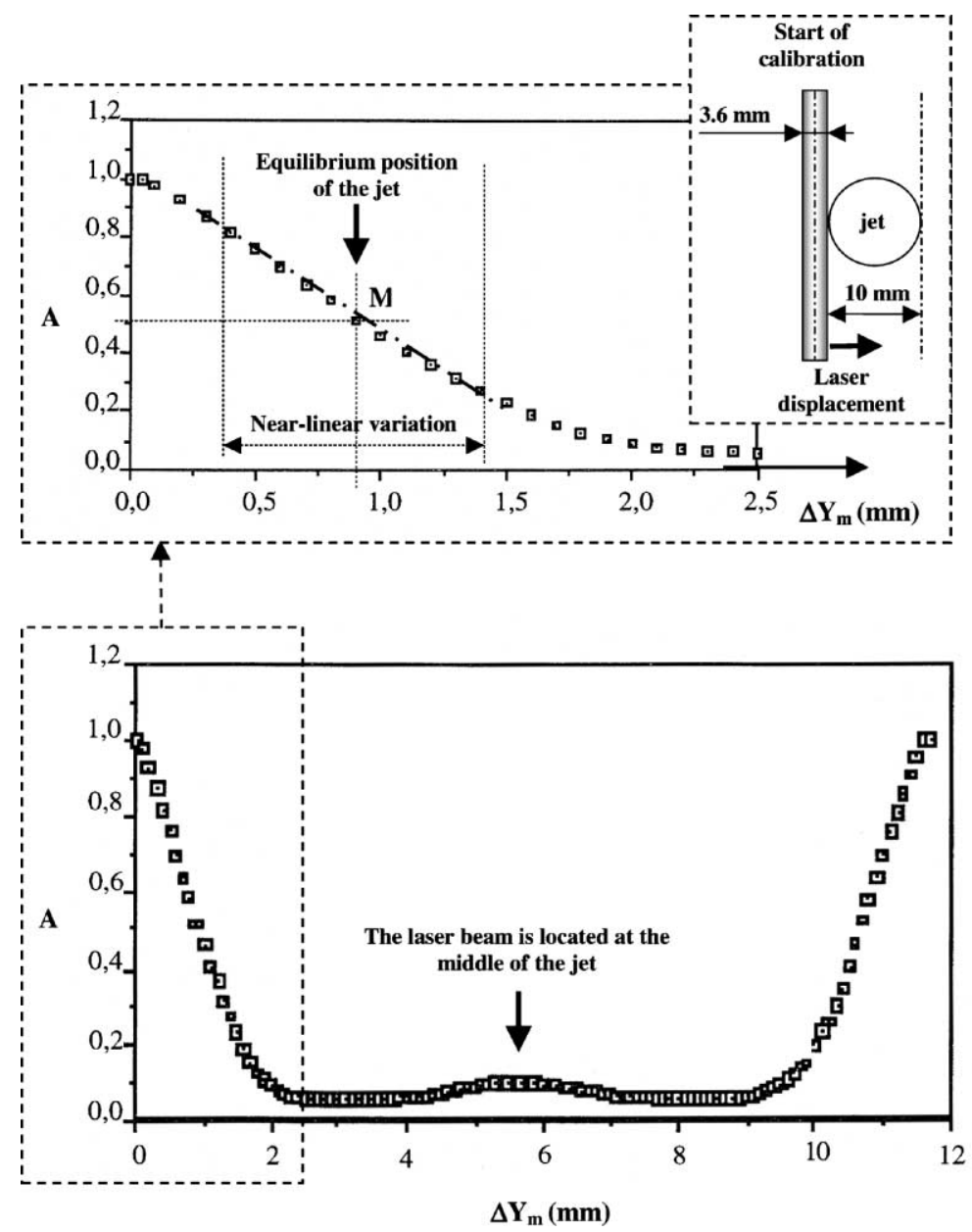

Fig. 5. The calibration curve. The symbols $A$ and $\Delta Y_{\mathrm{m}}$ denote, respectively, the non-dimensional optical signal delivered by the photodetector and the penetration depth of the laser beam within the liquid jet. The upper part of the figure is an enlargement of the quasi-linear area.

(Gaussian distribution). In other words, the edge of the laser beam is not marked. Therefore, we start the calibration with a small step and then increase to the current value $1 / 10 \mathrm{~mm}$. The last point for which the quantity $A$ is equal to unity is defined as the edge of the jet.

As displayed in Fig. 5, when half of the laser beam has penetrated the inside of the jet $(A=0.5)$, the abscissa $\Delta Y_{\mathrm{m}}$, further referred to as $\overline{\Delta Y_{\mathrm{m}}}$, is equal to about $0.9 \mathrm{~mm}$; this is the location of the point $\mathrm{M}$ in Fig. 5. This transversal location of the laser beam - in relation to the plunging jet - is found to be an equilibrium position, allowing the light absorption technique to apply well. The technique has been tested with three other media: a black tube, a bright tube (optically close to the plunging water jet when it is smooth) and a black screen. The three calibration curves thus obtained were found to be indistinguishable from the one obtained with a smooth water jet. More particularly, the curves exhibited invariably the same slope on a limited area, say, a quasi-linear area, always centred around the same point $\mathrm{M}$. Thus, at the vicinity of the point $\mathrm{M}$, the output 
voltage delivered by the set-up is always found quasi-linear whatever the nature of the liquid medium involved in the plunging jet is. Moreover, further analytical calculations allow to demonstrate that both the location of the point $\mathrm{M}, \overline{\Delta Y_{\mathrm{m}}}$, and the extent of the quasi-linear area, $L\left(\overline{\Delta Y_{\mathrm{m}}}\right)$, are always of the order of the laser spot radius (Appendix A). Making use of the quasilinearity of the optical technique, the following conclusions are worth to note:

1. any positive or negative displacement of the jet interface around the point $\mathrm{M}$ gives rise to the same change in the output voltage,

2. any small error on the localisation of the point $\mathrm{M}$ yields a negligible error in the output voltage, and finally,

3. the nature of a disturbance travelling on the jet does not make the technique to fail provided the amplitude of the disturbance remains smaller than the typical extent of the quasi-linear area on the calibration curve (Appendix A, H6).

The sensitivity of the optical technique, defined as $S=\partial\left(\Delta Y_{\mathrm{m}}\right) / \partial A$, is derived from a fitting approximation of the calibration curve. The sensitivity $S$ is found to be minimal but uniform around the optimal penetration length of the laser beam in the jet $\Delta Y_{\mathrm{m}}=\overline{\Delta Y_{\mathrm{m}}}$. This uniformity of the sensitivity $S$ thus observed over the quasi-linear area allows us to assess directly the experimental errors related to the light absorption technique. The error sources are identified either as small fluctuations of the laser emission, which yield AC voltages of the order of $0.03 \mathrm{~V}$ (assessed from an external Philips $50 \mathrm{MHz}$ scope), or as inaccuracies due to the sampling of the output voltage: the acquisition is performed on an ordinary A/D converter (12 bits on a voltage range of $\pm 5 \mathrm{~V}$, acquisition frequency: $250 \mathrm{KHz}$ ). These last inaccuracies are actually found to be negligible $\left(\sim 2.4 \times 10^{-3} \mathrm{~V}\right)$. The subsequent errors on the non-dimensional voltage $A$ and the interfacial displacement are then calculated in the linear area of the calibration curve:

$$
\delta \mathrm{A} \cong 0.003 \text { and } \delta\left[\Delta Y_{\mathrm{m}}\right] \cong 0.06 \mathrm{~mm} .
$$

The quasi-linear area makes also straightforward the transformation of the non-dimensional voltage $A(t)$ into a time-dependent signal $\Delta Y(t)(\mathrm{mm})$.

This signal usually expresses a spatially-averaged displacement of the jet interface because of the radial extent of the laser beam. Considering the notation on the schematic displayed in Fig. 6, the variable $\eta(t)$ denotes the local deformation of the jet interface. Therefore, the signal $\Delta Y(t)$ writes as follows:

$$
\Delta Y(t)=\overline{\Delta Y_{\mathrm{m}}}+\frac{1}{z_{2}-z_{1}} \int_{z_{1}}^{z_{2}} \eta(t) \mathrm{d} z,
$$

so that it has to be considered as a mean level of the interfacial agitation. Then, the smaller the cross-section of the laser beam is, the more local the measurement of the interface displacement is (see e.g. Fig. 9, Appendix A). Conversely, the larger the cross-section of the laser beam is, the better the detection of the interfacial disturbances of large amplitude is. At the same time, the displacement of the jet interface has to provide a change of light intensity large enough to fit the limited sensitivity of the photodetector. Consequently, for most of our experiments, the following agreement was found: the laser beam was expanded by means of a non-focal lenses arrangement to a diameter of $3.6 \mathrm{~mm}$ (jet diameter $d=10-16 \mathrm{~mm}$ ). 


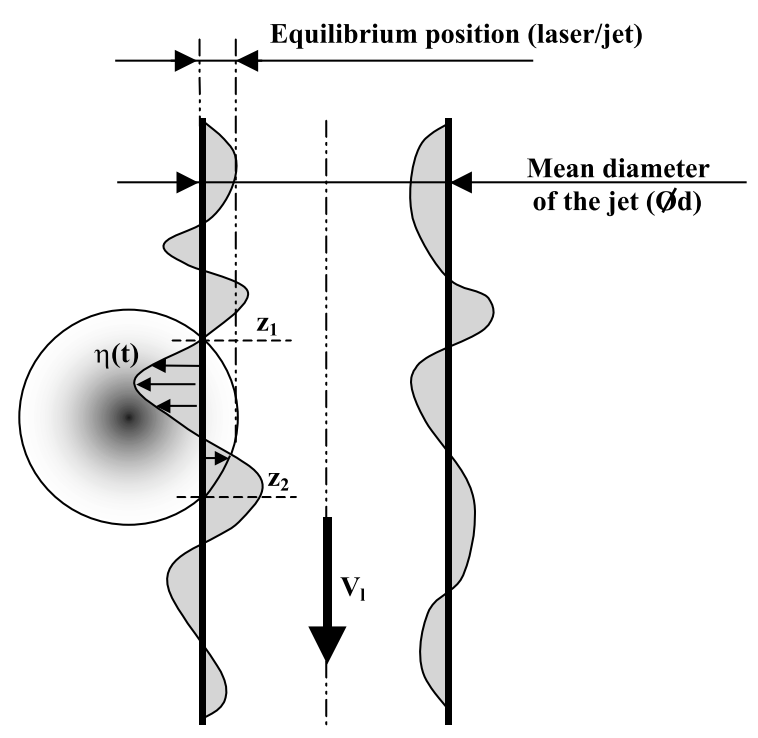

Fig. 6. The measurement of the averaged interfacial roughness.

More generally, an elementary modelling is drawn out in the appendix and helps to find out the interfacial disturbances (typical wavelength $\lambda$ and amplitude $\Omega$ ) which can be locally detected once the size of the laser spot is tuned. Few requirements are thus given which allow the user to apply conveniently the optical technique and to place conveniently the laser beam in relation to the jet.

\subsection{Limitations of the present optical technique}

If the plunging jet experiences an initial spray detachment mechanism (see e.g. Hoyt and Taylor, 1977), the optical technique under consideration is clearly not appropriate since it requires a location along the plunging jet where the interface keeps a significant degree of interfacial cohesion (at least near the nozzle).

Otherwise, a broad range of interfacial waves can be characterised, particularly, those which prefigure either breakup or the transition from a laminar to a turbulent regime: the helical (or sinuous) waves, the axisymmetric (or varicose) waves and the Kelvin-Helmholtz waves if the jet experiences a strong aerodynamic interaction with surrounding air. The analytical developments available in the appendix demonstrate that the technique exhibits a sufficient spatial resolution for any wave whose wavelength $\lambda$ is larger than $4 \pi \Omega$ where $\Omega$ denote the amplitude of the interfacial wave under consideration.

An undesirable situation clearly arises when the liquid jet is strongly turbulent all along its falling height: the limited spatial resolution of the optical technique does not allow for detecting small interfacial deformations due to turbulent structures in the inertial and Kolmogorov subranges (Wu and Faeth, 1995a). The technique is nevertheless hoped to apply to the diagnostic of the interfacial agitation driven by the energy-containing eddies since the mean shear within the liquid jet favours their deformation along the mean flow $(\lambda>\Omega)$.

A further improvement of the light absorption technique to a higher spatial resolution might be achieved when cutting off the incident laser light with a mask at jet width. Then, a complete 
modelling of the light absorption at the jet interface is much more delicate since the distribution of the light intensity is not easy to derive. A calibration may be necessary to get the local amplitude of the interfacial deformation. The typical wavelength has to be obtained by a more usual technique which consists in splitting the incoming laser sheet and to cross-correlate the two subsequent optical signals $A_{1}(t)$ and $A_{2}(t)$ along the jet.

\subsection{The (interfacial) dynamical roughness as a way to quantify air entrainment}

\subsubsection{Definition of the dynamical roughness}

Removing any numerical noise beforehand, the signal $\Delta Y(t)$ is time-differentiated. The subsequent signal $v^{\prime}(t)$ is defined as a characteristic fluctuating velocity of the jet interface. The dynamical (interfacial) roughness is then defined as the ratio $\varepsilon=\sigma_{\mathrm{e}} / V_{1}$, where $V_{1}$ and $\sigma_{\mathrm{e}}$ stand as, respectively, the jet velocity and the RMS of the fluctuating velocity $v^{\prime}(t): \sigma_{\mathrm{e}}=\left(\overline{\mathrm{v}^{\prime 2}}\right)^{1 / 2}$. This definition of the dynamical roughness $\varepsilon$, original as far as we know from the fairly scarce literature, can be physically justified taking the example of an interfacial instability $\eta(t)$ travelling along the jet at the celerity $c$. To simplify the demonstration, $\eta(t)$ is supposed to be a monochromatic harmonic wave at least at the vicinity of the laser spot (see Appendix A). Hence, the signal $v^{\prime}(t)$ writes as,

$$
v^{\prime}=\frac{\mathrm{d} \eta(t)}{\mathrm{d} t}=-\frac{2 \pi \Omega \mathrm{c}}{\lambda} \cos \frac{2 \pi}{\lambda}(z-c t) .
$$

The symbols $\Omega, \lambda, z$ and $t$ denote, respectively, the local amplitude and the local wavelength of the disturbance, the distance along the jet and time. Moreover, the intrinsic celerity of the interfacial wave $c_{\mathrm{i}}$ (consider a referential moving with the mean flow) can be usually supposed to be negligible with respect to the jet velocity $V_{1}\left(c=c_{\mathrm{i}}+V_{1} \cong V_{1}\right)$. As a consequence, the interfacial roughness simplifies as,

$$
\varepsilon=\frac{\sigma_{\mathrm{e}}}{V_{1}}=\frac{\pi \sqrt{2} \Omega}{\lambda}
$$

since

$$
\sigma_{\mathrm{e}}=\sqrt{\overline{\left(v^{\prime}\right)^{2}}}=\frac{2 \pi \Omega}{\lambda} c \sqrt{\lim _{T \rightarrow \infty}\left[\frac{1}{T} \int_{T}\left[\cos ^{2} \frac{2 \pi}{\lambda}(z-c t)\right] \mathrm{d} t\right]}=\frac{2 \pi \Omega}{\lambda} c \frac{1}{\sqrt{2}} \sim \frac{\sqrt{2} \pi \Omega}{\lambda} V_{1} .
$$

The interfacial roughness $\varepsilon$, as it is defined in this paper, turns out to be proportional to the magnitude of the interfacial deformation $\eta(t)$ and inversely proportional to the local wavelength of $\eta(t)$. Since the Weber number of the jet $W e_{\mathrm{j}}$ takes into account the typical jet velocity $V_{1}$, it is therefore consistent to find that $\varepsilon$, as an additional non-dimensional number, is not dependent on $V_{1}$. These considerations are consistent with the natural physical intuition that, all things being equal, the more the magnitude of the disturbance is and the smallest the wavelength is, the more efficient the air entrapment at the impinging point is.

As a matter of fact, an other definition of the interfacial roughness was put forward by McKeogh and Ervine (1981). They introduced the concept of (geometrical) interfacial roughness defined as the ratio of the typical magnitude of the disturbances to the mean radius of the jet. It 
seems to us that such a definition suffers somewhat from a lack of generality since it does not take into account the dependence of the amount of air carryunder on the wavelengths (or integral scales) that characterise the interfacial disturbances on the jet.

\subsubsection{Experimental uncertainties}

Error sources of the present technique are assessed around the equilibrium position $\overline{\Delta Y_{\mathrm{m}}}$. The definition of the dynamical roughness, $\varepsilon=\sigma_{\mathrm{e}} / V_{1}$, yields the following experimental uncertainty: $\delta \varepsilon=\delta \sigma_{\mathrm{e}} / V_{1}+\sigma_{\mathrm{e}} \delta V_{1} / V_{1}^{2}$. The mean jet velocity $V_{1}$, compared to the RMS of the fluctuating velocity, $\sigma_{\mathrm{e}}$, is a negligible source of errors especially in the present study where use is made of an external loop providing a very steady liquid flow rate. Technical details on this external loop are furnished in a companion paper (El Hammoumi et al., in press). During the calibration procedure, it was clearly observed that a smooth water jet, a black or a bright tube and a black screen all yield invariably the same signals $v^{\prime}(t)$ with the same broad-band noise. In these conditions, the experimental uncertainty on the RMS of the fluctuating velocity was measured as $\delta \sigma_{\mathrm{e}} \cong 10^{-7}$ $\mathrm{m} \mathrm{s}^{-1}$.

\subsubsection{An evidence of the dependence of the air entrainment on the initial dynamical roughness}

The measurement of $\varepsilon$ is expected to be a reliable indicator of the air entrainment efficiency if the turbulent agitation of the free-surface of the receiving pool plays a negligible role to the air entrapment. To make the correlation between $\varepsilon$ and the amount of air carryunder evident, an experimental set-up has been carried out which allows us to excite a plunging jet at a given level of hydrodynamical noise. This experimental set-up of which a schematic is shown in Fig. 7 involves a vibrator monitored from an external power supply and linked to a rigid vertical plate. This plate, whose motion is transversal and sinusoidal, is plunged within a tank filled with a constant volume of Lobelia oil or water. An external loop, not represented in Fig. 7, allows to keep the height of

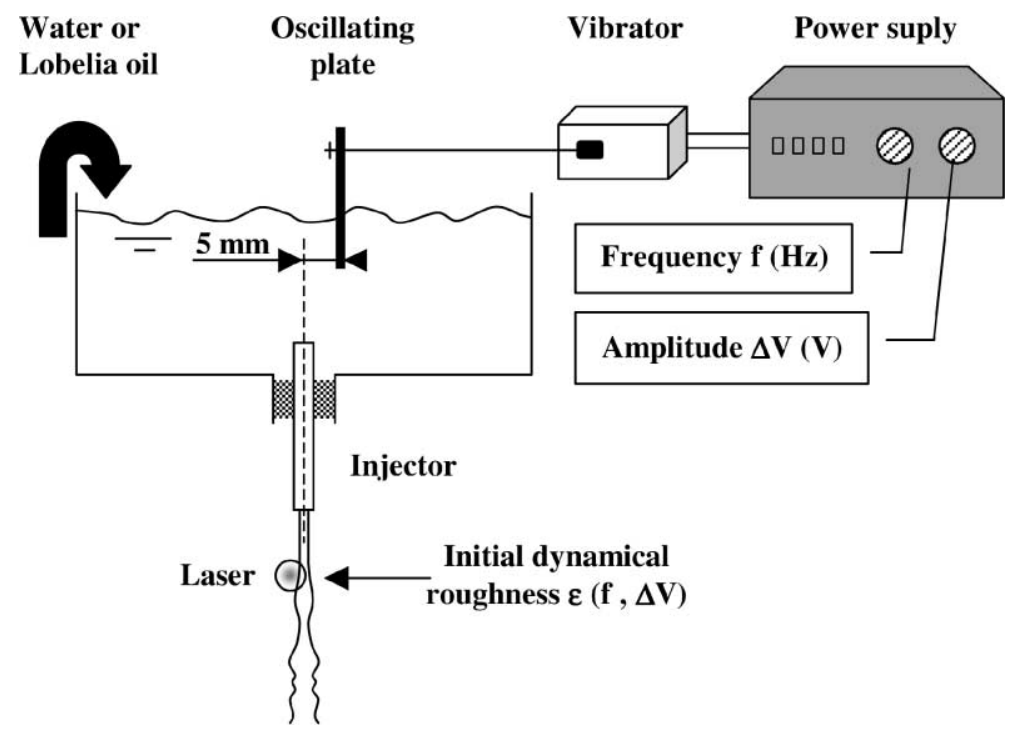

Fig. 7. Overall schematic of the experimental facility devoted to supply the plunging jet with hydrodynamical noise. 
Table 1

Characteristics of the plunging jets involved in the present study (recall that $R e_{j}=W e_{j}^{0.5} / Z$ )

\begin{tabular}{lll}
\hline & Laminar jet (Lobelia oil) & Turbulent jet (water) \\
\hline$W e_{j}$ & 861.57 & 554.76 \\
$F r$ & 14.97 & 49.57 \\
$Z$ & $43.75 \times 10^{-3}$ & $1.251 \times 10^{-3}$ \\
$\widetilde{H}$ & 20.36 & 32.22 \\
\hline
\end{tabular}

this liquid bath constant, and therefore, to keep the mean velocity of the jet, $V_{1}$, very steady. A (turbulent) jet of water and a (laminar) jet of oil (Lobelia TB15), are supplied from an ordinary cylindrical tube used as nozzle (diameter $\Phi=14 \mathrm{~mm}$ ). The tube is long enough for the jet to be fully-developed at the exit. Table 1 provides the values of the non-dimensional numbers involved in the present study. The flow rate $\Phi_{\mathrm{a}}$ and therefore, the Weber number of entrainment, $W e_{\mathrm{n}}$, was measured with an experimental facility of which details are provided in a companion paper (El Hammoumi et al., in press).

We expect that the vertical oscillating plate controls the magnitude and the spectral content of the hydrodynamical noise supplied to the plunging jet. The mean position of the plate in the supplier tank ( $5 \mathrm{~mm}$ away from the axis of the injector) has been optimised in order to give the maximum level of the initial interfacial roughness $\varepsilon$ of the jet near the nozzle exit. Thus, the dependence of $\varepsilon$ on the parameters of the power supply, namely the frequency $f$ and the amplitude $\Delta V$ of the AC voltage applied to the vibrator, is investigated. The time-dependence of the velocity fluctuation $v^{\prime}(t)$ on the voltage $\Delta V$ is studied by way of Fourier-transforms and cross-correlations. The spectral contents of $v^{\prime}(t)$ and $\Delta V$ share common energetic harmonics only when the jet is laminar. When the jet becomes turbulent, the system $\{$ injector + jet $\}$ behaves as a complex filter which makes disappear any correlation between the forcing and the interfacial agitation of the jet.

When the plunging jet is laminar, the dependence of the Weber of entrainment $W e_{\mathrm{n}}$ on $\epsilon$ is found monotonic and close to a linear progression (Fig. 8a). When the jet becomes turbulent,
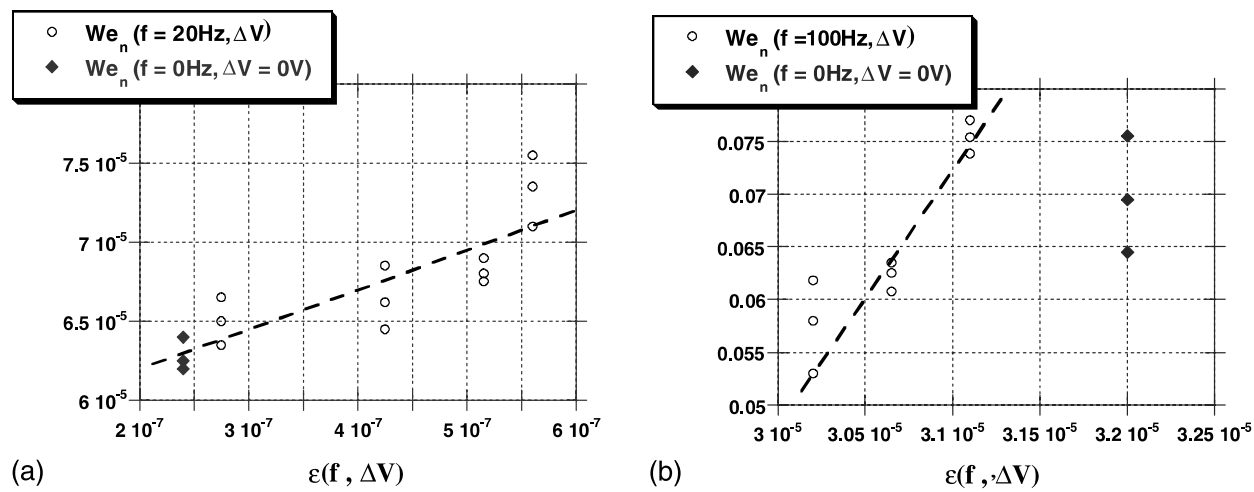

Fig. 8. Dependence of the Weber number of entrainment, $W e_{\mathrm{n}}$, on the initial dynamical roughness $\varepsilon$ (circles points). The amplitude of the forcing, $\Delta V$, is varied whereas its frequency $f$ and the jet velocity $V_{1}$ are kept constant (see also Table 1 ). The diamond points are performed with both the frequency and the amplitude of the oscillating motion set to zero. (a) Laminar jet (Lobelia oil, $f=20 \mathrm{~Hz}, V_{1}=1.43 \mathrm{~m} \mathrm{~s}^{-1}$ ). (b) Turbulent jet (water, $f=100 \mathrm{~Hz}, V_{1}=2.04 \mathrm{~m} \mathrm{~s}^{-1}$ ). 
a large variation applied either to the level of amplitude or to the frequency of the oscillations leads to a small variation in $\varepsilon$ : an increase of $80 \%$ on the frequency or an increase of $85 \%$ on the amplitude of the oscillations yields invariably a $3 \%$ variation of $\varepsilon$. But, at the same time, small though any variation of $\varepsilon$ is (a few percents), the increase in the amount of air carryunder $\Phi_{\mathrm{a}}$ is found to be dramatic when the jet is turbulent (Fig. 8b). The diamond points plotted in Fig. 8a and $b$ represent a set of measurements performed when no oscillating motion is present in the tank (vertical plate at rest). The data are aligned each others except when the jet is turbulent (Fig. 8a and $b$ ), then the absence of any forcing drive to a larger interfacial roughness here referred to as $\varepsilon(0,0)$ (frequency and magnitude of the forcing tuned to zero). The manner by which the mechanical energy of the oscillating plate is transferred to the initial flow conditions of the turbulent jet remains difficult to understand. Moreover, a rather bad reproducibility is observed on the measurement of the entrainment Weber number, which, in turn, is surprisingly low with regard to the level of $\varepsilon(0,0)$.

- A first explanation which comes to mind is connected to the following measurement artefact. Assuming the interfacial disturbances exhibit a large amplitude, it could be thought that the light absorption technique is characterised by an insufficient spatial resolution. And subsequently, the measurements should not take into account the large disturbances responsible for an efficient air entrapment at the impinging point. But here, a close attention was given to place the laser beam near the exit of the injector so as to characterise interfacial disturbances when they exhibit a convenient magnitude (Appendix A).

- The second explanation - that we put forward - is based on the distinction between the scenarii \#II and \#III. We think that the free-surface of the receiving pool is submitted to a level of turbulent agitation high enough to entrap a non-negligible amount of air (scenario \#III). The measurement of $\varepsilon$ do not take into account this jet momentum-driven agitation at the free-surface of the receiving pool.

Despite this last point, it can be claimed without any doubt that whatever the nature of the jet (laminar or turbulent) is, the air entrainment and subsequently, the number $W e_{\mathrm{n}}$, exhibit a monotonic dependence on the initial roughness $\varepsilon$. A slope, $P_{\mathrm{e}}$, defined as,

$$
P_{\mathrm{e}}=\frac{\partial W e_{\mathrm{n}}}{\partial \varepsilon}
$$

can be inferred from a linear fitting of the $W e_{\mathrm{n}}$ measurements (see e.g. the dotted lines displayed in Fig. 8a and b). The subsequent calculated value of $P_{\mathrm{e}}$ is equal to 24 or $2.4 \times 10^{4}$ depending on whether the jet is, respectively, laminar or turbulent. The initial interfacial roughness of the jet at the exit of the injector can thus be considered as an essential control parameter of the air entrainment process.

\section{Final comment}

An analysis of the literature on the air entrainment by plunging jets has been performed. The great diversity of the physical mechanisms involved in the air entrainment process have been 
considered and discussed. This diversity can be partially handled through the dependence of the entrainment Weber number on several non-dimensional numbers, namely: the Weber number, the Froude number, the Öhnesorge number, the non-dimensional falling height, the ratio of the liquid density to the gas carryunder density, the ratio of viscosities and the initial dynamical roughness. This quantity must not be confused with the geometrical roughness introduced by McKeogh and Ervine (1981) which characterises the jet just before its impact and which is given a too exclusive role.

The majority of plunging jet configurations can be classified according to three generic scenarii (initially introduced by McKeogh and Ervine (1981)), to which we add a fourth scenario, theoretically studied by Lezzi and Prosperetti (1991). These scenarii and their differentiation are the object of detailed comments. Up to now, the fourth scenario has never been the subject of any detailed experimental investigation. And yet, it stands as a very interesting reappraisal of the widespread idea according to which the air entrainment would be mostly driven by air entrapment processes controlled by pure potential flows.

When the liquid jet is weakly-viscous, it turns out that none single model is able to represent with fair agreement the whole of the available experimental data. The explanation mainly relies on the badly-controlled hydrodynamical conditions delivered to the plunging jet by the injector. This paper demonstrates that any residual hydrodynamical noise available at the nozzle can increase drastically the ability of the plunging jet to entrap air; the magnitude of the initial hydrodynamical noise applied to the jet is correlated to the level of initial dynamical jet roughness $\varepsilon$.

Conversely, measuring $\varepsilon$ stands as a convenient tool to design optimally the internal geometry of an injector. To set accurately the level of the hydrodynamical noise delivered to the jet, a way to do is to promote more or less a relaminarisation of the jet under the effect of a favourable pressure gradient inside the injector. This can be achieved taking advantage of an annular contraction whose aperture is finely tuned (El Hammoumi et al., 1999). It is worth to note that such a technology could also help to provide an experimental insight into the air entrainment scenario modelled by Lezzi and Prosperetti (1991). This scenario involves a high-speed liquid jet which remains smooth even at a large Reynolds number of the jet $\left(R e_{\mathrm{j}} \sim 10^{4}\right.$ or more). The viscosity of the air carryunder plays also a key-role since the annular air sheet dragged down by the liquid jet beneath the free surface of the pool becomes unstable under the effect of the shear.

The paper presents also a handy optical technique which allows us to measure $\varepsilon$ and then to assess the magnitude of the hydrodynamical noise delivered to the jet: a quantity of interest for any spatial stability analysis. This optical technique is based on the absorption of the light energy supplied by a laser beam which matches the edge of the plunging jet and thus measures its dynamical roughness $\varepsilon$. It is demonstrated that the response of this light absorption technique is quasi-linear provided the laser emission is of $\mathrm{TEM}_{00}$-type. For such a laser, few analytical developments led under peculiar requirements (Appendix A) allow for the complete characterisation of travelling long waves. If the laser is neither perfectly Gaussian nor perfectly axisymmetric, the light absorption technique still applies well but a full calibration of the optical set-up has to be performed with the help of a perfectly smooth jet. The incident laser spot can be expanded or focused at will depending on the nature of the interfacial waves to detect (long gravity waves or capillary waves) or depending on the vertical position of the laser spot with respect to the jet. This particular ability allows us to tune the spatial resolution of the laser spot from about $100 \mu \mathrm{m}$, typically, the capillary scale, to about $1 \mathrm{~cm}$. Clearly, when turbulence of the plunging jet is fully- 
established, the experimental technique here proposed is no longer appropriate since it doesn't exhibit a spatial resolution high enough to detect the interfacial instabilities related to the inertial or Kolmogorov sub-ranges. One nevertheless expects the optical technique remains sensitive to interfacial waves originated from the energy-containing eddies.

Finally, the measurement of the dynamical roughness is hoped to help future studies on nearsurface turbulence.

\section{Acknowledgements}

The authors are indebted to the SERAC company (France) for its decisive financial support. The authors wish to thank also the valuable technical contribution of G. Picaud.

\section{Appendix A. Modelling of the light absorption technique}

The existence of a quasi-linear area on the calibration curve, which is proved to be very useful because of the linear dependence of the optical signal on the penetration length $\Delta Y_{\mathrm{m}}$, is directly dependent on the Gaussian axisymmetry of the light intensity distribution. Moreover, when perfect Gaussian axisymmetry holds, it turns out that the accurate measurement of the interfacial waves which develop on the jet (frequency, geometry of the disturbance/local amplitude, wavelength) can be performed. Under these conditions, the analytical developments which follow allow to predict the calibration curve for the light absorption technique depicted in Fig. 9. The example of the interfacial deformation due to a travelling axisymmetric wave is given as an illustration in Fig. 9 while the meaning of all the notations involved in the following is illustrated. The symbols $Y_{\mathrm{m}}$ and $\theta$ denote, respectively,

- the transversal location of the jet interface with respect to the laser beam centre,

- and the angular deviation between the horizontal [ON]-axis of the laser spot and one radius [ON'] (see Fig. 9).

\section{A.1. Calculation of the calibration curve}

The hatched area displayed in the enlarged area of Fig. 9 (bottom rectangle) is the part of the laser spot hidden by the liquid jet; the light energy $I_{\mathrm{abs}}$ thus absorbed writes as

$$
I_{\text {abs }}\left(Y_{\mathrm{m}}\right)=2 \int_{\theta_{\mathrm{m}}}^{\pi / 2}\left(\int_{Y_{\mathrm{m}} / \sin \theta}^{R_{\mathrm{f}}} I(r) r \mathrm{~d} r\right) \mathrm{d} \theta \quad \text { with } I(r)=I_{0} \mathrm{e}^{-2 r^{2} / w^{2}} .
$$

This expression of $I_{\text {abs }}$ is based on the following assumptions:

H1. The interfacial deformation of the jet - at the scale of the laser cross-section-is supposed to be negligibly small ( $Y_{\mathrm{m}}$ or $\Delta Y_{\mathrm{m}}$ are uniformly constant). This requirement, always fulfilled 


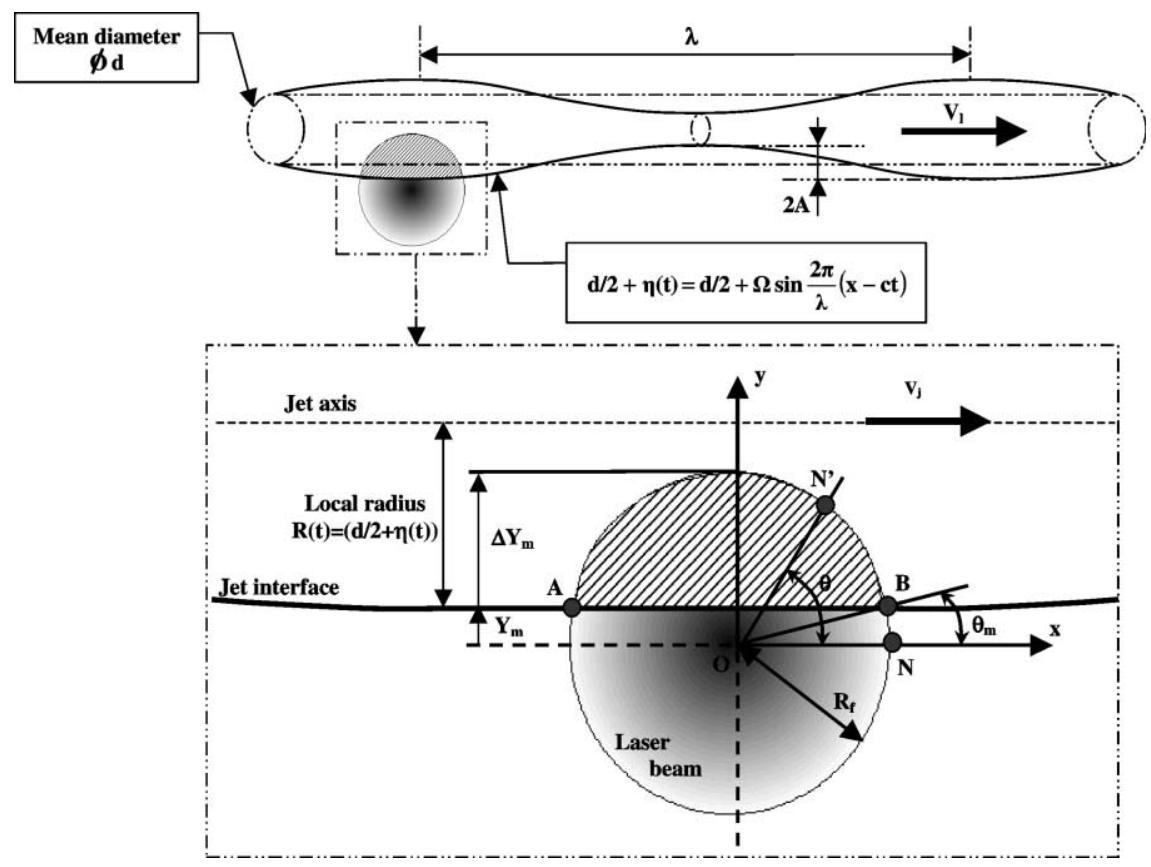

Fig. 9. The optical technique as sketched and the significance of all the notations involved in the modelling. A varicose wave is chosen as an example.

during the calibration procedure, demands, otherwise, any travelling wave on the jet to exhibit a characteristic wavelength much larger than the typical radial extent of the laser spot. That situation is studied in the last section of this appendix.

$\mathrm{H} 2$. The variable $Y_{\mathrm{m}}$ has to remain strictly positive $\left(Y_{\mathrm{m}}>0\right)$ for the integral to keep sense. Anticipating on the following, this hypothesis is not a restrictive one since the absorbed light intensity distribution exhibits a symmetry (Fig. 10). As a consequence, the calculated calibration curve can be merely continued by reflection to the point M (Fig. 10) in order to take account of the light absorption on the whole of the laser spot. This reflection can be equivalently understood as a plane symmetry to the [ON]-axis (Fig. 9).

The expression of the Gaussian intensity distribution $I(r)$ is defined from the so-called diameter, $w$, of the laser beam. Recall that the diameter $w$ is a local variable which changes with a distance $z$ apart the laser source, decreasing first, and then, increasing from the waist.

H3. Here, we choose to define the typical radius $R_{\mathrm{f}}$ of the laser spot as $R_{\mathrm{f}}=w / 2$.

The non-deflected part of the laser beam is reflected by the mirror and is finally received by the photodetector. Subsequently, the light energy actually measured, say $I_{\mathrm{ph}}$, stands as the difference between the total input intensity of the laser $I_{\text {tot }}$ and $I_{\mathrm{abs}}$ where,

$$
I_{\mathrm{tot}}=2 \int_{-\pi / 2}^{\pi / 2}\left(\int_{0}^{R_{\mathrm{f}}} I(r) r \mathrm{~d} r\right) \mathrm{d} \theta=2 \pi I_{0} R_{\mathrm{f}}^{2}\left(1-\mathrm{e}^{-(1 / 2)}\right) .
$$




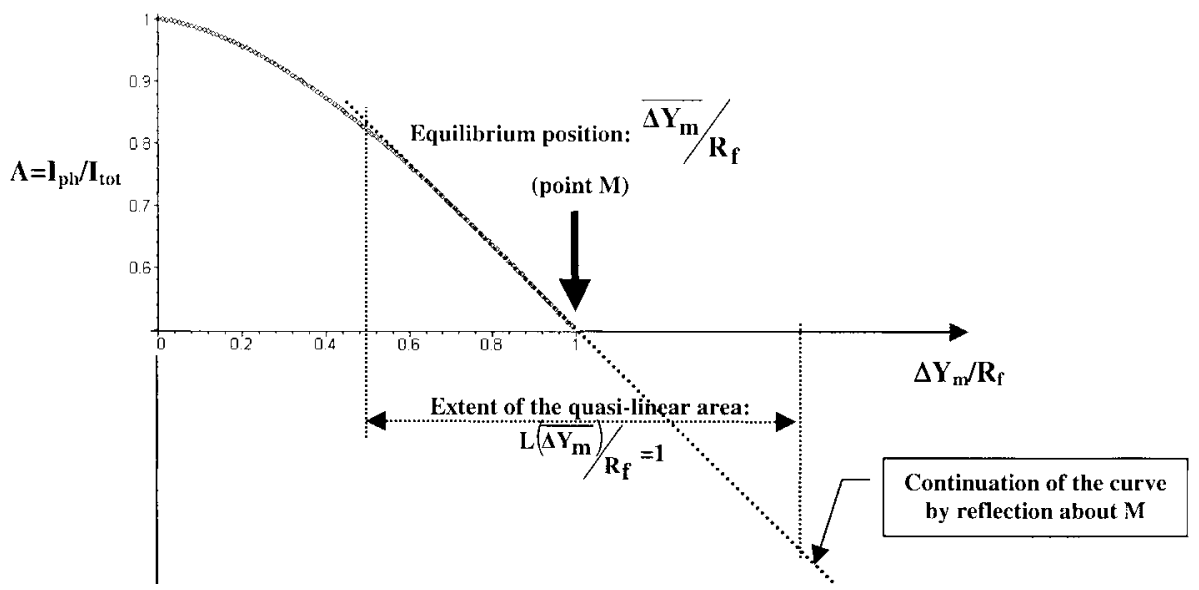

Fig. 10. The non-dimensional calibration curve as computed. Quasi-linearity arises when the penetration length $\Delta Y_{\mathrm{m}}$ is equal to half the laser spot radius $R_{\mathrm{f}}$; then, the laser spot centre and the jet interface are separated each other a distance $R_{\mathrm{f}} / 2$ apart.

In order to characterise from a general manner both the equilibrium position of the laser spot with regard to the jet interface and the extent of the quasi-linear area, it may be of interest to write the non-dimensional light intensity $A=I_{\text {abs }} / I_{\text {tot }}$-which is nothing but the optical signal—as a quantity dependent on the non-dimensional variable: $a=Y_{\mathrm{m}} / R_{\mathrm{f}}$. Subsequently, the non-dimensional penetration length of the laser spot into the jet and the expression for the angular deviation $\theta_{\mathrm{m}}$ can be defined as, respectively,

$$
(1-\mathrm{a})=\frac{\Delta Y_{\mathrm{m}}}{R_{\mathrm{f}}} \quad \text { and } \quad \sin \theta_{\mathrm{m}}=\frac{Y_{\mathrm{m}}}{R_{\mathrm{f}}} .
$$

Few elementary calculations (including the change of variable, $u=\left[\Delta Y_{m} / \sin \theta R_{\mathrm{f}}\right]^{2}$ ) drive to the following expression for the optical signal $A=I_{\mathrm{ph}} / I_{\mathrm{tot}}$ :

$$
A=\frac{I_{\mathrm{ph}}}{I_{\mathrm{tot}}}(a)=1+\frac{(1 / 2-\arcsin (a) / \pi) \mathrm{e}^{-1 / 2}}{\left(1-\mathrm{e}^{-1 / 2}\right)}+\frac{a}{2 \pi\left(1-\mathrm{e}^{-1 / 2}\right)} \int_{1}^{a^{2}} \frac{\mathrm{e}^{-u / 2}}{u \sqrt{\left(u-a^{2}\right)}} \mathrm{d} u \text {. }
$$

It is then straightforward to calculate numerically the following calibration curve (Fig. 10).

Thus, both the equilibrium position of the laser beam $\overline{\Delta Y_{\mathrm{m}}}$ and the typical extent of the quasilinear area $L \overline{\left(\Delta Y_{\mathrm{m}}\right)}$ are found to be of the order of $R_{\mathrm{f}}$ (taking account of the symmetry to the horizontal axis of the laser spot).

\section{A.2. Application of the optical technique to the detection of waves}

As far as travelling interfacial waves are concerned, it may be of interest to establish a criteria about the smallest wavelength $\lambda$ of the wave such that the optical technique still applies conveniently without any loss of spatial resolution. As pictured in Fig. 9, the wave under consideration 
here is assumed monochromatic and harmonic in such a way that the local radius of the jet writes as:

$$
R(t)=\frac{d}{2}+\eta(t) \quad \text { where } \eta(t)=\Omega \sin \frac{2 \pi}{\lambda}(x-c t) .
$$

The variables $t, d, \Omega$ and $c$ denote, respectively, the time, the averaged diameter of the jet, the amplitude and the celerity of the travelling wave with respect to the (fixed) laser spot referential. Here again, the local radius $R(t)$ is supposed to be uniform at the level of the laser spot:

$\mathrm{H} 4$. The wavelength of the harmonic interfacial deformation is much larger than the laser spot radius $R_{\mathrm{f}}$ so that the penetration of the laser spot in the jet can be thought of as uniform. The centre of the laser spot and the edge of the jet are located a distance $Y_{\mathrm{m}}$ apart, with $Y_{\mathrm{m}}(t)=$ $\overline{Y_{\mathrm{m}}}-\eta(t)$ where the variable $\overline{Y_{\mathrm{m}}}$ is defined as follows: $\overline{Y_{\mathrm{m}}}=R_{\mathrm{f}}-\overline{\Delta Y_{\mathrm{m}}}$.

For the spatial resolution of the laser spot to be sufficient:

$\mathrm{H} 5$. The maximum slope along the jet interface, $\Omega 2 \pi / \lambda$, has to remain lower than the ratio $L\left(\overline{\Delta Y_{m}}\right) / 2 \sqrt{\overline{\Delta Y_{m}}\left(2 R_{\mathrm{f}}-\overline{\Delta Y_{m}}\right)}$ (ratio of the quasi-linear area extent to the distance AB, see Fig. 9) For the linearity of the optical technique to hold:

H6. The peak to peak amplitude of the interfacial wave has to remain lower than the typical extent of the quasi-linear area $\overline{L\left(\Delta Y_{\mathrm{m}}\right)}$.

Taking account of the general findings deduced from the calculated calibration curve, the former hypothesis $\mathrm{H} 5$ and $\mathrm{H} 6$ write, respectively, as follows:

$$
\Omega \frac{2 \pi}{\lambda}<\frac{1}{2} \quad \text { and } \quad \Omega<\frac{R_{\mathrm{f}}}{2} .
$$

These requirements makes the optical technique involved in this paper particularly relevant to the detection of long waves $(\lambda>4 \pi \Omega)$.

Further analytical calculations show that any long wave can be faithfully followed provided it exhibits an amplitude $\Omega$ small enough (the exact criterion is given hereafter). The light energy can be time-differentiated exactly in the same way as it is done when calculating numerically the interfacial roughness $\varepsilon$; the subsequent expression writes:

$$
\frac{\mathrm{d} I_{\mathrm{ph}}}{\mathrm{d} t}=\frac{-\mathrm{d} I_{\mathrm{abs}}}{\mathrm{d} t}=-\frac{\mathrm{d}}{\mathrm{d} t}\left[2 \int_{\theta_{\mathrm{m}}}^{\pi / 2}\left(\int_{Y_{\mathrm{m}} / \sin \theta}^{R_{\mathrm{f}}} I_{0} \mathrm{e}^{-2 r^{2} / \mathrm{w}^{2}} r \mathrm{~d} r\right) \mathrm{d} \theta\right],
$$

or alternatively,

$$
\frac{\mathrm{d} I_{\mathrm{ph}}}{\mathrm{d} t}=-2 I_{0} R_{\mathrm{f}}^{2}\left\{\mathrm{e}^{-1 / 2} \frac{\mathrm{d} \theta_{\mathrm{m}}}{\mathrm{d} t}+I(t)\right\} \quad \text { with } I(t)=\frac{\mathrm{d}}{\mathrm{d} t}\left[\int_{\theta_{\mathrm{m}}}^{\pi / 2} \mathrm{e}^{-1 / 2\left(\frac{Y_{\mathrm{m}}}{R_{\mathrm{f}} \sin \theta}\right)^{2}} \mathrm{~d} \theta\right] .
$$

Taking account of the equality $\sin \theta_{\mathrm{m}}=Y_{\mathrm{m}} / R_{\mathrm{f}}$ and making use of the changes of variable $v=\sin \theta$ and $u=\sin \theta_{\mathrm{m}}$, it is also possible to write the former time-derivative $I(t)$ as

$$
\left.I(t)=-\frac{\mathrm{d} \theta_{\mathrm{m}}}{\mathrm{dt}} \quad \frac{\mathrm{e}^{-1 / 2}}{\cos \theta_{\mathrm{m}}}+\frac{1}{2} \sin 2 \theta_{\mathrm{m}} \int_{\mathrm{u}}^{1} \frac{\mathrm{e}^{-\mathrm{u}^{2} / 2 v^{2}}}{v^{2} \sqrt{1-v^{2}}} \mathrm{~d} v\right)
$$


where use is made of the Leibnitz rule. As a consequence, the time-derivative of the non-dimensional optical signal $A(t)$ can be simplified to the following formula ${ }^{4}$,

$$
\frac{1}{I_{\text {tot }}} \frac{\mathrm{d} I_{\mathrm{ph}}}{\mathrm{d} t}(\mathrm{t})=\frac{-\mathrm{d} \eta / \mathrm{d} t}{\pi R_{\mathrm{f}}\left(\mathrm{e}^{\frac{1}{2}}-1\right)}\left[\left(1+\sqrt{\frac{\pi}{2}} \mathrm{e}^{\frac{1}{2}\left(1-\mathrm{a}^{2}\right)}\right) \operatorname{erf}\left(\sqrt{\frac{1-\mathrm{a}^{2}}{2}}\right)-\sqrt{1-\mathrm{a}^{2}}\right],
$$

where use has been made of a truncated series expansion of $\theta_{\mathrm{m}}$, with respect to the variable $\eta(t) / R_{\mathrm{f}}$, about the point $\overline{Y_{\mathrm{m}}} / R_{\mathrm{f}}$, up to second order. This last approximation relies on the assumption that:

H7. Only a monochromatic harmonic wave, $\eta(t)$, is supposed to be responsible for the interfacial deformation of the jet, the magnitude of which, $\Omega$, being supposed much lower than the laser spot radius $R_{\mathrm{f}}$.

More generally, these calculations show which kind of waves can be completely characterised by the optical technique. These waves have to fulfill the requirements $\mathrm{H} 4-\mathrm{H} 6$. They are long gravity waves or capillary waves and subsequently, the size $R_{\mathrm{f}}$ of the laser spot has to be, respectively, enlarged or reduced.

\section{References}

Bin, A.K., 1984. Air entrainment by plunging liquid jets. Proceedings of the symposium on scale effects in modelling hydraulic structures, Paper 5.5, pp. 1-6.

Bin, A.K., 1988. Gas entrainment by plunging liquid jets. VDI-Forschungsh 648/88, 1-36.

Bin, A.K., 1993. Gas entrainment by plunging liquid jets. Chem. Engng. Sci. 48 (21), 3585-3630.

Bonetto, F., Lahey, R.T., 1993. An experimental study on air carryunder due to a plunging liquid jet. Int. J. Multiphase Flow 19 (2), 281-294.

Burgess, J.M., Molly, N.A., Mc Carthy, M.J., 1972. A note on plunging liquid jet reactor. Chem. Engng. Sci. 27, 442445.

Chanson, H., Cummings, P., 1992. Aeration of the ocean due to plunging waves. Research report, No. CE142, Department of Civil Engineering, University of Queensland, Australia.

Chanson, H., 1992. Air entrainment in chutes and spillways. Research report, No. CE133, Department of Civil Engineering, University of Queensland, Australia.

Cossali, G.E., Coghe, A., 1993. A new laser based technique for instability growth rate evaluation in liquid jets. Exp. Fluids 14, 233-240.

Dabiri, D., 1999. Free-surface roughness correlations with the near-surface turbulence. Free Surface Turbulence and Bubbly Flows Workshop, ONR Workshop, University of California at San Diego.

El Hammoumi, M., Achard, J.-L., Davoust, L., 1999. Mesure de la rugosité d'un jet par atténuation lumineuse, application à l'optimisation d'un injecteur. XIV $V^{\text {ieme }}$ Congrès Français de Mécanique, Toulouse, France.

El Hammoumi, M., Achard, J.-L., Davoust, L., in press. Measurements of air entrainment by vertical plunging liquid jets. Exp. Fluids, in press.

\footnotetext{
${ }^{4}$ Several successive changes of variables allow us to derive the integral involved in the expression of $I(t)$ as follows:
}

$\int_{\mathrm{u}}^{1} \frac{\mathrm{e}^{-\mathrm{u}^{2} / 2 v^{2}}}{v^{2} \sqrt{1-v^{2}}} \mathrm{~d} v=\frac{1}{2} \sqrt{\frac{2 \pi}{u^{2}}} \mathrm{e}^{-u^{2} / 2} \operatorname{erf}\left(\sqrt{\frac{1}{2}\left(1-u^{2}\right)}\right)$. 
Ervine, D.A., Falvey, H.T., 1987. Behaviour of turbulent water jets in the atmosphere and in plunge pools. Proc. Instn Civ. Engrs., Water Engineering Group, Paper 913683 (Part 2), 295-314.

Fenn, R.W., Middleman, S., 1969. Newtonian jet stability: the role of air resistance. AIChE J. 15, 379-383.

Grant, R.P., Middleman, S., 1966. Newtonian jet stability. AIChE J. 12, 669-678.

Hoyt, J.W., Taylor, J.J., 1977. Waves on water jets. J. Fluid Mech. 83, 119-127.

Iciek, J., 1982. The hydrodynamics of a free liquid jet and their influence on direct contact: heat transfer 1. Int. J. Multiphase Flow 8 (3), 239-249.

Kusabiraki, D., Niki, H., Yamagiwa, K., Ohkawa, A., 1990a. Gas entrainment rate and flow pattern of vertical plunging liquid jets. Can. J. Chem. Engng. 68, 893-903.

Kusabiraki, D., Murota, M., Ohno, S., Yamagiwa, K., Yasuda, M., Ohkawa, A., 1990b. Gas entrainment rate and flow pattern in a plunging liquid jet aeration system using inclined nozzles. J. Chem. Engng. Japan 23, 704-710.

Lafrance, P., 1977. The breakup length of turbulent liquid jets. J. Fluids Engng., Trans. ASME, 414-415.

Lara, P., 1979. Onset of air entrainment for water jet impinging vertically on water surface. Chem. Engng. Sci. 34, $1164-1165$.

Lezzi, A.M., Prosperetti, A., 1991. The stability of an air film in a liquid flow. J. Fluid Mech. 226, 319-347.

Lin, T.J., Donnelly, D.G., 1966. Gas bubble entrainment by plunging laminar liquid jets. AIChE J. 12 (3), 563-571.

Mc Carthy, M.J., Henderson, J.B., Molloy, N.A., 1970. In: Proc. Chemeca 1970 Conf., Australia, vol. 2. Butterworths, London, pp. 86-100.

McKeogh, E.J., Ervine, D.A., 1981. Air entrainment rate and diffusion pattern of plunging liquid jets. Chem. Engng. Sci. 36, 1161-1172.

Mouza, A.A., Vlachos, N.A., Paras, S.V., Karabelas, A.J., 2000. Measurement of liquid film thickness using a laser light absorption method. Exp. Fluids 28, 355-359.

Oguz, H.N., 1998. The role of surface disturbances in the entrainment of bubbles by a liquid jet. J. Fluid Mech. 372, 189-212.

Ohl, C.D., Oguz, H.N., Prosperetti, A., 2000. Mechanism of air entrainment by a disturbed liquid jet. Phys. Fluid. 12 (7), 1710-1714.

Phinney, R.E., 1973. Stability of a laminar viscous jet. The influence of an ambiant gas. Phys. Fluids 16 (2), 193-196.

Prosperetti, A., Oguz, H.N., 1997. Air entrainment upon liquid impact. Phil. Trans. R. Soc. Lond. A 355, 491-506.

Reitz, R.D., Braco, F.V., 1982. Mechanism of atomization of a liquid jet. Phys. Fluids 25 (10), 1730-1742.

Sene, K.J., 1988. Air entrainment by plunging jets. Chem. Engng. Sci. 43, 2615-2623.

Sterling, A.M., Sleicher, C.A., 1975. The instability of capillary jets. J. Fluid Mech. 68 (3), 477-495.

Torpey, P.A., 1989. A nonlinear theory for describing the propagation of disturbances on a capillary jet. Phys. Fluid. A $1(4), 661-671$.

Van de Sande, E., Smith, M., 1973. Surface entrainment of air by high velocity water jets. Chem. Engng. Sci. 28, 11611168.

Van de Sande, E., Smith, M., 1976. Jet break-up and air entrainment by low velocity turbulent water jets. Chem. Engng. Sci. 31, 219-224.

Weber, C., 1931. Zum Zerfall eines Flussigkeitsstrahles. Z. Angew. Math. Mech. 11, 136-154.

Wu, P.K., Faeth, G.M., 1995a. Onset and end of drop formation along the surface of turbulent liquid jets in still gases. Phys. Fluids A. 7 (11), 2915-2917.

Wu, P.K., Miranda, R.F., Faeth, G.M., 1995b. Effects of initial flow conditions on primary breakup of nonturbulent and turbulent liquid jets. Atom. Sprays 5, 175-196.

Zhu, Y., Oguz, H.N., Prosperetti, A., 2000. On the mechanism of air entrainment by liquid jets at a free surface. J. Fluid Mech. 404, 151-177. 